\title{
Stress-induced martensitic transformations in NiTi and NiTi-TiC composites investigated by neutron diffraction
}

\author{
R. Vaidyanathan ${ }^{\text {a }}$, M.A.M. Bourke ${ }^{\mathrm{b}}$, D.C. Dunand ${ }^{\mathrm{a}, *}$ \\ ${ }^{a}$ Department of Materials Science and Engineering, Massachusetts Institute of Technology, Cambridge, MA 02139, USA \\ ${ }^{\mathrm{b}}$ LANSCE/MST, Los Alamos National Laboratory, Los Alamos, NM 87545, USA
}

\begin{abstract}
Superelastic NiTi (51.0 at.\% Ni) specimens reinforced with 0,10 and 20 vol.\% TiC particles were deformed under uniaxial compression while neutron diffraction spectra were collected. The experiments yielded in-situ measurements of the thermoelastic stress-induced transformation. The evolution of austenite/martensite phase fractions and of elastic strains in the reinforcing TiC particles and the austenite matrix were obtained by Rietveld refinement [1] during the loading cycle as the austenite transforms to martensite (and its subsequent back transformation during unloading). Phase fractions and strains are discussed in terms of load transfer in composites where the matrix undergoes a stress-induced phase transformation. (C) 1999 Elsevier Science S.A. All rights reserved.
\end{abstract}

Keywords: Shape-memory; Superelasticity; Metal matrix composites; Neutron diffraction; Rietveld refinement; Phase transformation

\section{Introduction}

Unlike metal matrix composites where the matrix flow occurs primarily by slip, little is known about composites where the matrix deforms by alternative deformation mechanisms (e.g. twinning or stress-induced transformations). The intermetallic NiTi with near-equiatomic composition may deform by a reversible, thermoelastic transformation between a hightemperature, cubic (B2) parent austenitic phase and a low-temperature, monoclinic (B19') martensitic phase [2]. Dunand et al. [3-7] have systematically characterized such NiTi-based shape-memory composites where a martensitic NiTi matrix deforms by twinning in the presence of TiC particles. However, to the best of our knowledge, no study exists on the mechanical behavior of NiTi-based composites where an austenitic matrix deforms by forming stress-induced martensite in the presence of a reinforcing ceramic phase. We recently demonstrated the ability to observe stress-induced

\footnotetext{
* Corresponding author. Present address: Department of Materials Science and Engineering, Northwestern University, 2225 N. Campus Drive, Evanston, IL 60208, USA. Tel.: + 1-847-4915370; fax: + 1847-4676573.

E-mail address: dunand@nwu.edu (D.C. Dunand)
}

transformations in NiTi by subjecting mechanicallyloaded NiTi samples to neutron diffraction [8]. In the present paper, this technique is used to investigate the evolution of martensite fractions and discrete phase strains in superelastic NiTi and $\mathrm{NiTi}-\mathrm{TiC}$ composites under compression loading.

\section{Experimental procedures}

Sample characterization and fabrication by hot-isostatic-pressing of prealloyed NiTi $(51.0$ at. $\% \mathrm{Ni})$ powders are described elsewhere [9]. Specimens of three compositions were produced: (a) unreinforced NiTi (b) NiTi with 10 vol.\% TiC and (c) NiTi with 20 vol.\% TiC, hereafter referred to as NiTi-0TiC, NiTi-10TiC and NiTi-20TiC. Cylindrical samples $(10 \mathrm{~mm}$ in diameter and $24 \mathrm{~mm}$ in length) were solutionized in titanium-gettered flowing argon at $1000^{\circ} \mathrm{C}$ for $1 \mathrm{~h}$, oil-quenched to room temperature, annealed in air at $400^{\circ} \mathrm{C}$ for one hour and quenched in ice-water. An average grain-size of $20 \mu \mathrm{m}$ was measured for all three compositions, which all exhibited a stable austenitic phase at room temperature. No transformation to martensite was detected upon cooling with liquid helium to temperatures as low as $-253^{\circ} \mathrm{C}$. The stable 
nature of the austenite phase is beyond the scope of the present discussion and is discussed elsewhere [9].

Details concerning the experimental setup, which is only summarized here, can be found in $[6,10]$. Neutron diffraction measurements were performed in 'time-offlight' mode using the neutron powder diffractometer (NPD) at the pulsed neutron source at Los Alamos Neutron Science Center (LANSCE). One sample of each composition was loaded in uniaxial compression at room temperature while neutron diffraction spectra were acquired in three scattering geometries. The loading axis formed an angle of $45^{\circ}$ with the incident neutron beam, allowing measurements for which the scattering vectors were parallel and perpendicular to the loading axis. Since the incident beam is polychromatic, by choosing an appropriate bank of detectors, diffraction from planes parallel or perpendicular to the loading direction can be investigated. A third detector (at an angle of $32^{\circ}$ from the incident beam) provided a measurement in a back-scattering geometry. An extensometer attached to the cylindrical compression specimens recorded the macroscopic strain during the experiments.

Fig. 1 shows the macroscopic stress-strain curves of the samples indicating the stress levels at which neutron spectra were obtained over a period of 6-8 h (all compressive stresses and strains are reported as positive values). These load-unload cycles were obtained after training the sample twice to $625 \mathrm{MPa}$ at a cross-head speed of $3 \mathrm{~mm} \mathrm{~min}^{-1}$ in order to stabilize the transformation by removing any instabilities or heterogeneities

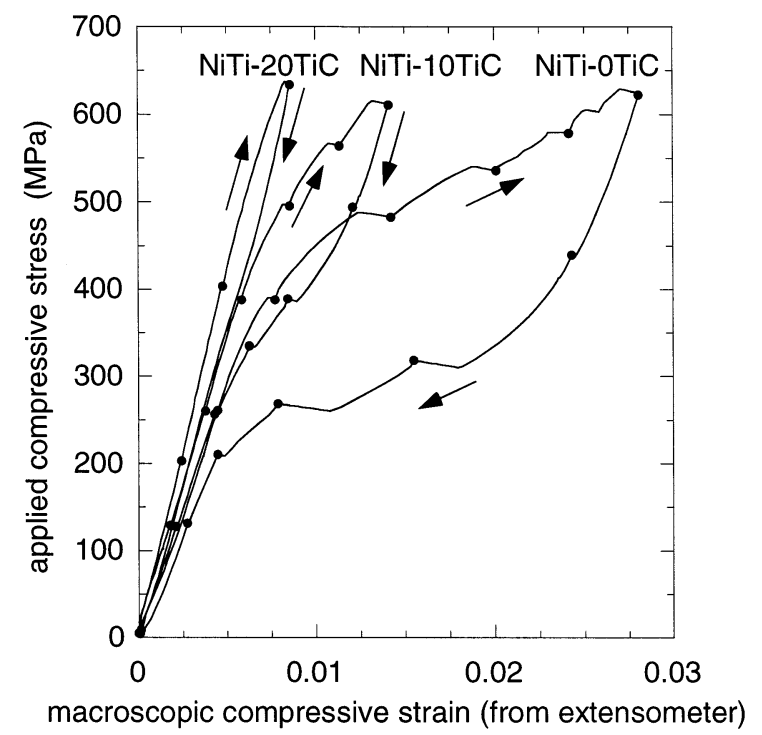

Fig. 1. Curves of applied compressive stress versus macroscopic compressive strain measured by extensometry for $\mathrm{NiTi}-0 \mathrm{TiC}, \mathrm{NiTi}-$ 10TiC and NiTi-20TiC. The dots indicate the stress levels at which neutron diffraction spectra were obtained. that may exist. At the higher measurement stress levels, the samples continued to accumulate strain before reaching (within a few minutes) the strain levels marked with black dots. This effect is attributed to the relatively high loading rate which did not allow sufficient time for transformation enthalpy dissipation [11-13]. Since the transformation is thermoelastic, a strain is produced in the sample upon equilibrating to ambient temperature. This phenomenon had no significant effect on the neutron measurements due to the long diffraction time.

\section{Neutron diffraction data analysis}

By performing a Rietveld refinement [1] using the LANSCE code GSAS [14], the analysis encompasses all of the reflections and represents the overall deformation of the polycrystal. In the Rietveld refinement method, a mathematical model is developed that calculates an intensity, $Y_{\mathrm{c}}$, at every point in the spectra, i.e.:

$$
Y_{\mathrm{c}}=Y_{\mathrm{b}}+\sum_{h} S K F_{h}^{2} P\left(\Delta T_{\mathrm{h}}\right)
$$

where the first term, $Y_{\mathrm{b}}$, is the background intensity and the second term is the Bragg scattering containing a scale factor, $S$, a correction factor, $K$, a structure factor, $F_{\mathrm{h}}$, and a profile function, $P\left(\Delta T_{\mathrm{h}}\right)$, as determined by the displacement, $\Delta T_{\mathrm{h}}$, of the profile point from the reflection position. The refinement procedure varies various parameters related to phase volume fractions and atom positions until the calculated spectrum matches the measured spectrum in a least squares fit. In a conventional Rietveld refinement the data is assumed to originate from an untextured powder. However, the deformed austenite (despite being untextured at the start and finish of the test) develops a reversible texture that correlates with the austenite/martensite ratio $[8,9]$. This, along with the evolving texture in the martensite, is accommodated in the refinement by a generalized harmonic description of the texture $[15,16]$ within the correction factor $K$.

The other aspect of the refinements that warrants special attention is the strain. Since loading was uniaxial, the 'macroscopic' strain differs parallel and perpendicular to the load direction. The lattice plane $(h k l)$ dependent strains are also typically highly anisotropic. An average isotropic strain is used to capture these effects empirically, shifting the individual lattice reflections according to changes in the lattice parameter. Thus, in the present work, changes in the lattice parameter are the basis for reporting strains from neutron diffraction measurements. A more rigorous analysis of the strain and texture will be presented in a future publication [17]. 


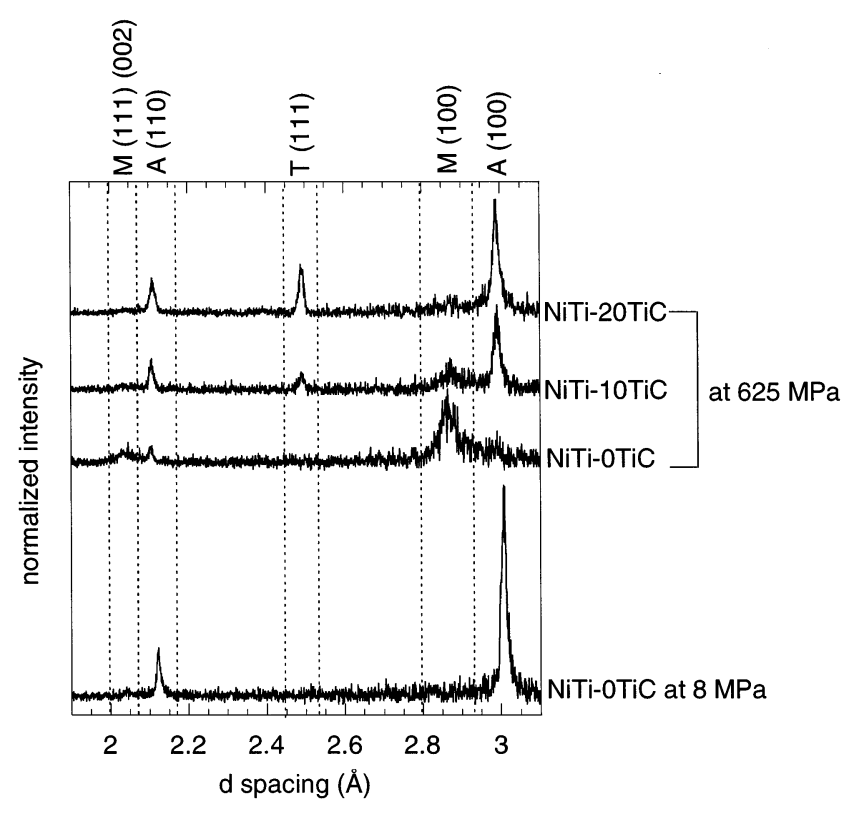

Fig. 2. Section of normalized neutron diffraction spectra from NiTi0TiC, NiTi-10TiC and NiTi-20TiC at $625 \mathrm{MPa}$ compared to the spectrum of NiTi-0TiC at $8 \mathrm{MPa}$ with the austenite (A), martensite (M) and $\mathrm{TiC}(\mathrm{T})$ peaks identified. A nominal stress of $8 \mathrm{MPa}$ was used as the 'no load' condition to hold the specimen horizontally in the rig.

\section{Results and discussion}

In agreement with $[3,18,19]$, no reaction was observed by optical microscopy between the NiTi matrix and the $\mathrm{TiC}$ particles, which were almost perfectly stoichiometric with a composition of $49.8 \pm 0.1$ at. $\% \mathrm{C}$ (as determined by combustion analysis with infrared detection). Given the inert nature of the $\mathrm{TiC}$ and the identical initial materials and processing routes for all samples, the matrix chemical composition can be assumed constant in all samples, suggesting that the differences in mechanical behavior observed in Fig. 1 are not due to chemical effects.

Fig. 2 compares a section of the normalized neutron spectra for lattice planes perpendicular to the loading direction. Qualitative comparison of the martensite (100) peaks at $625 \mathrm{MPa}$ shows that the amount of martensite decreases with increasing TiC content. From a Rietveld refinement of diffraction data from all three detectors, the volume fraction of martensite in $\mathrm{NiTi}-$ $0 \mathrm{TiC}$ and $\mathrm{NiTi}-10 \mathrm{TiC}$ was quantified as a function of the applied stress, as shown in Fig. 3. For NiTi-20TiC, the volume fraction of martensite present was too small ( $<5$ vol. $\%$ at maximum load) for the refinement to converge. The difference in macroscopic strain-stress curves observed between NiTi-0TiC, NiTi-10TiC and NiTi-20TiC (Fig. 1) may thus be attributed to the differences in volume fractions of stress-induced martensite due to the presence of the reinforcing particles.
Table 1 shows the Young's moduli for the three specimens as determined from extensometer data. The values are obtained from a linear fit to the region prior to the onset of non-linearity in the curves in Fig. 1. Table 1 also shows predictions for the moduli using stiffness tensor values from ultrasonic measurements of NiTi single crystals from [20]. The polycrystal Young's moduli are predicted as a single-crystal average using Hashin-Shtrikman [21] upper and lower bounds for cubic polycrystals. The upper and lower bounds for austenite are tight and predict moduli of 74.4 and 75.4 $\mathrm{GPa}$, respectively. The Voigt and Reuss average suggested by Hill [22] gives a modulus of $74.5 \mathrm{GPa}$. Using slightly different single-crystal data from [23], the predicted values differ by only a few GPa. The values predicted for the composite are obtained from the Eshelby equivalent inclusion method outlined in [6,24] with elastic $\mathrm{TiC}$ data from [25]. In predicting an average modulus for the composite, coefficient of thermal expansion (CTE) mismatch stresses between austenite and $\mathrm{TiC}$ are neglected because of their low values: the average matrix tensile stress is $24.9 \mathrm{MPa}$ in NiTi10TiC and 49.1 MPa in NiTi-20TiC from the above method, assuming a temperature drop of $731 \mathrm{~K}$ and CTE values from [26,27].

Table 1 shows that there is a significant discrepancy between the predicted and measured moduli. This discrepancy cannot be explained by texture in the austenite since Rietveld refinements indicate no texture at these low load levels. The predictions assume purely elastic contributions to the modulus while the extensometer may measure inelastic contributions, (e.g., strains due to the stress-induced transformation). Since

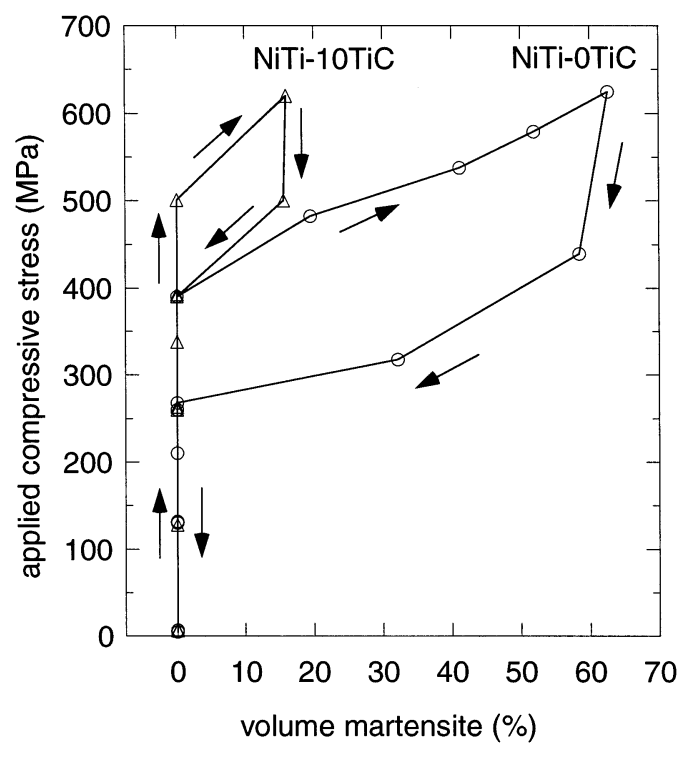

Fig. 3. Volume fraction of martensite obtained from Rietveld refinements as a function of the applied compressive stress in NiTi-0TiC and NiTi-10TiC. 
Table 1

Young's moduli (GPa) of NiTi-0TiC, NiTi-10TiC and NiTi-20TiC as determined from extensometry and as predicted from literature values; discrepancies suggest non-elastic contributions to the extensometer moduli

\begin{tabular}{lccl}
\hline & Extensometer measurement $(\mathrm{GPa})$ & Theoretical prediction $(\mathrm{GPa})$ & Method of prediction \\
\hline NiTi-0TiC & $50 \pm 2$ & 75.4 & Hashin-Shtrikman [21] \\
& & 74.5 & Hill average [22] \\
NiTi-10TiC & $58 \pm 2$ & 87.3 & Eshelby [24] \\
NiTi-20TiC & $74 \pm 2$ & 102.1 & Eshelby [24] \\
\hline
\end{tabular}

the strains associated by the austenite-martensite transformation are large, a small volume fraction of austenite transforming to martensite almost linearly with increasing stress can account for the observed differences. Assuming that favorable orientations of austenite transform to martensite at these low stresses (e.g. producing a compressive strain of $5.2 \%$ in the $<011>$ direction with reference to the parent phase vector basis [28]), a mere 1 vol.\% of martensite is needed every 78 MPa to explain the difference in Young's moduli for NiTi-0TiC in Table 1. These small volume fractions are below the experimental sensitivity limit. Despite additional elastic phase mismatch in the composites, even smaller volume fractions of martensite are needed to account for the moduli differences in the composites.

Fig. 4 shows a plot of the applied stress vs. the elastic lattice strain in the austenite phase of NiTi-0TiC for planes perpendicular to the loading direction, as obtained from the Rietveld analysis during loading and unloading. The slope of the line, corresponding to an average modulus, is $74.5 \mathrm{GPa}$, which is in good agreement with either of the predicted values (Table 1). However, the modulus of the austenite phase measured by this method would be expected to change at higher load levels due to: (i) load transfer to the stress-induced martensite which exhibits different elastic constants; (ii) transformation mismatch stresses between the two phases, and (iii) the evolving texture in the austenite. The latter effect results from the strong texture qualitatively observed in individual spectra of the stress-induced martensite and the resulting austenite texture, given the unique lattice correspondence that exists between the austenite and the martensite phases. However Fig. 4 shows that these three effects do not cause any significant non-linearity in the stress-strain response of austenite in either loading or unloading, despite the fact that more martensite exists during unloading than loading for the same stress level due to the hysteresis shown in Fig. 3. This suggests that the non-linear effects either cancel each other or have negligible magnitude.

Fig. 5 for NiTi-10TiC and Fig. 6 for NiTi-20TiC show the elastic lattice strains determined from neutron diffraction in the austenite and $\mathrm{TiC}$ phases and the corresponding predictions from Eshelby's elastic theory. Values obtained from the single-crystal averaging methods for the austenite were used for the matrix in the predictions, in the light of its behavior in Fig. 4, while elastic constants from [25] were used for TiC. The Eshelby prediction also includes the initial thermal mismatch stresses calculated above (residual strains at zero applied stress). The strains obtained from the diffraction data are referenced to these residual stresses in the no load condition. Figs. 5 and 6 show reasonable agreement between elastic theory and experimental data.

Agreement in Fig. 6 is expected for NiTi-20TiC because little martensite is formed, so that the composite deforms mostly elastically. On the other hand, NiTi-10TiC shows significant amounts of stress-induced martensite at high applied stresses (Fig. 3) which could lead to additional mismatch with the TiC particles and thus deviation from the elastic response in Fig. 5 , as observed in plastically deforming aluminum composites [29]. The lack of such plastic load-transfer suggests that the austenite/martensite matrix effectively accommodates the mismatch arising due to the large transformation strains associated with the superelastic deformation. This may be attributed to the self-accom-

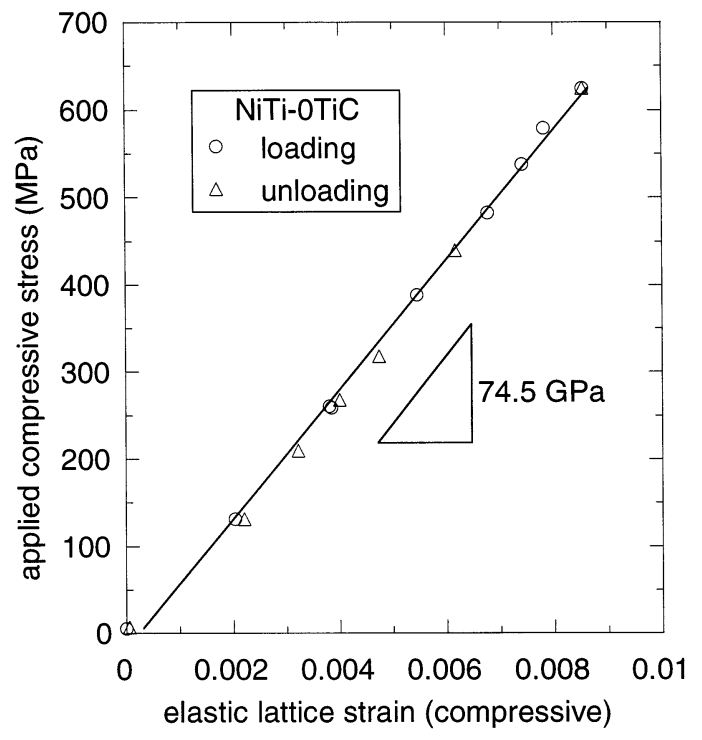

Fig. 4. Applied compressive stress versus elastic lattice strain (compressive) in the austenite phase of NiTi-0TiC, as obtained from Rietveld refinements of diffraction spectra. 


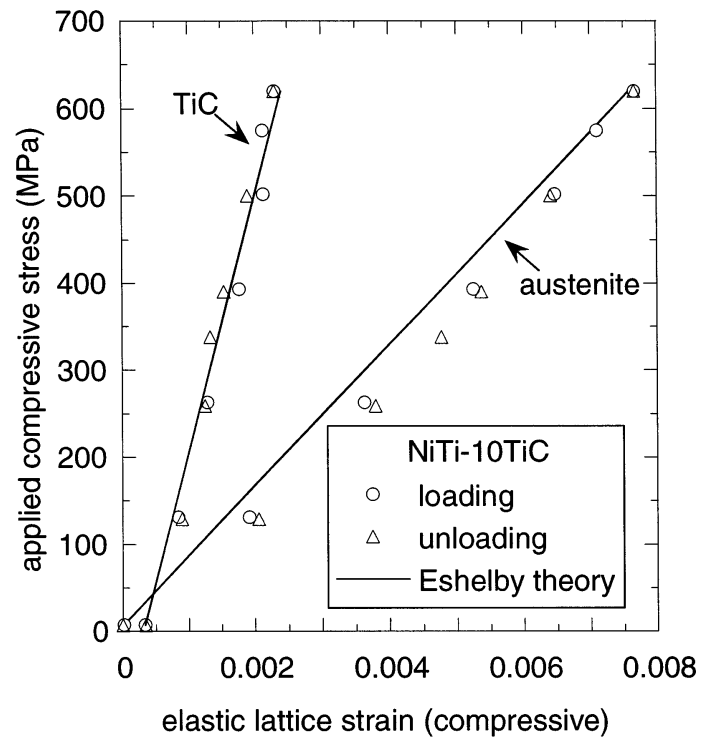

Fig. 5. Applied compressive stress versus elastic lattice strain (compressive) in the austenite and $\mathrm{TiC}$ phases of $\mathrm{NiTi}-10 \mathrm{TiC}$, as obtained from Rietveld refinements of diffraction spectra and as predicted by Eshelby's theory.

modating nature of the transformation, wherein certain variants can preferentially form to minimize mismatch between matrix and reinforcement, as also observed in shape-memory $\mathrm{NiTi}-\mathrm{TiC}$ composites deforming by martensite twinning [4]. A companion publication [9] investigating in detail the texture, phase fraction and strain evolution in these materials at higher applied stresses will be published separately.

\section{Conclusions}

Rietveld refinements of neutron diffraction spectra have been used to elucidate stress-induced transformations in superelastic NiTi containing 0, 10 and 20 vol.\% TiC particles. The generalized spherical harmonics texture formulation provides a determination of the austenite and martensite phase fractions evolving in the matrix. The volume fraction of martensite formed at any given applied stress decreases with increasing $\mathrm{TiC}$ content. Good agreement is observed between Eshelby's elastic theory and phase strains in the transforming matrix and the elastic $\mathrm{TiC}$ reinforcement, suggesting that the self-accommodating nature of the stress-induced martensite minimizes the transformation mismatch with the $\mathrm{TiC}$ particles.

\section{Acknowledgements}

The Manuel Lujan Jr. Neutron Scattering Center is a national user facility funded by the US DOE (Contract

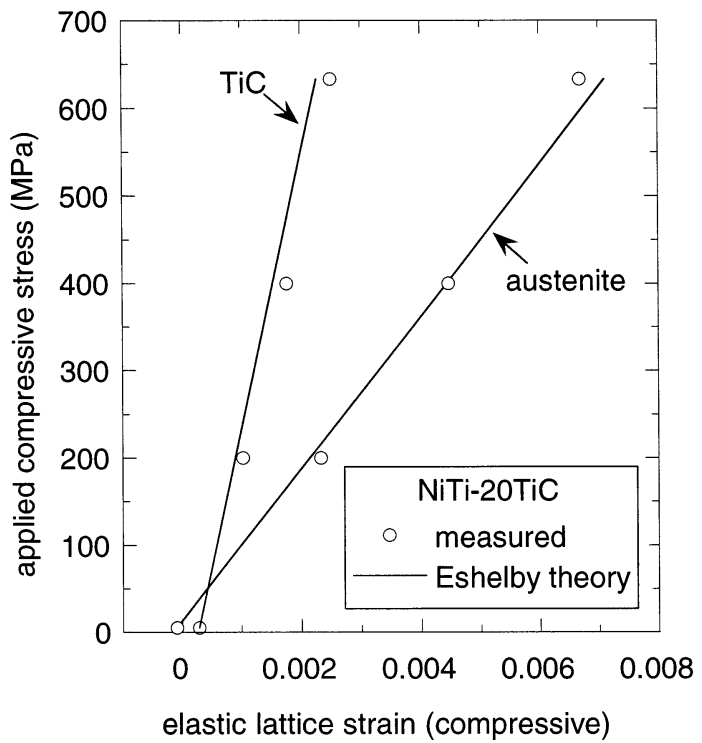

Fig. 6. Applied compressive stress vs. elastic lattice strain (compressive) in the austenite and $\mathrm{TiC}$ phases of $\mathrm{NiTi}-20 \mathrm{TiC}$, as obtained from Rietveld refinements of diffraction spectra and as predicted by Eshelby's theory.

No. W-7405-ENG-36) and by Defense Programs. RV and DCD acknowledge the support of Daimler Benz $A G$ in the form of a research grant. The authors also thank Dr. H. Pettermann and Dr. M.R. Daymond for helpful discussions.

\section{References}

[1] H.M. Rietveld, J. Appl. Crystallogr. 2 (1969) 65.

[2] T.W. Duerig, K.N. Melton, D. Stoeckel, C.M. Wayman, Engineering Aspects of Shape Memory Alloys, Butterworth-Heinemann, London, 1990.

[3] D. Mari, D.C. Dunand, Metall. Mater. Trans. 26A (1995) 2833.

[4] K.L. Fukami-Ushiro, D.C. Dunand, Metall. Mater. Trans. 27A (1996) 183.

[5] K.L. Fukami-Ushiro, D. Mari, D.C. Dunand, Metall. Mater. Trans. 27A (1996) 193.

[6] D.C. Dunand, D. Mari, M.A.M. Bourke, J.A. Roberts, Metall. Mater. Trans. 27A (1996) 2820.

[7] D.C. Dunand, K.L. Fukami-Ushiro, D. Mari, J.A. Roberts, M.A.M. Bourke, in: E.P. George, R. Gotthardt, K. Otsuka, S. Trolier-McKinstry, M. Wun-Fogle (Eds.), Materials for Smart Systems II, Materials Research Society, Pittsburg, 1996, p. 131.

[8] M.A.M. Bourke, R. Vaidyanathan, D.C. Dunand, Appl. Phys. Lett. 69 (1996) 2477.

[9] R. Vaidyanathan, M.A.M. Bourke, D.C. Dunand, Acta Mat. (1999) in press.

[10] N. Shi, M.A.M. Bourke, J.A. Roberts, J.E. Allison, Metall. Mater. Trans. 28A (1997) 2741.

[11] P.H. Leo, T.W. Shield, O.P. Bruno, Acta Metall. Mater. 41 (1993) 2477.

[12] S. Miyazaki, K. Otsuka, Y. Suzuki, Scripta Metall. 15 (1981) 287.

[13] P.G. McCormick, Y. Liu, Mat. Sci. Eng. A167 (1993) 51.

[14] A.C. Larson, R.B. Von Dreele, General Structure Analysis System (GSAS), Los Alamos National Laboratory LAUR 8-748, 1986. 
[15] H.J. Bunge, Texture Analysis in Materials Science, Butterworth-Heinemann, London, 1982.

[16] R.B. Von Dreele, J. Appl. Cryst. 30 (1997) 517.

[17] R. Vaidyanathan, M.A.M. Bourke, D.C. Dunand, J. Appl. Phys. (1999) in press.

[18] T.M. Poletika, S.N. Kulkov, V.E. Panin, Poroshkova Metallurgiya 7 (247) (1983) 54.

[19] S.N. Kulkov, T.M. Poletika, A.Y. Chukhlomin, V.E. Panin, Poroshkovaya Metallurgiya 8 (260) (1984) 88.

[20] T.M. Brill, S. Mittelbach, W. Assmus, M. Muellner, B. Luethi, J. Phys. Condens. Matter 3 (1991) 9621.

[21] Z. Hashin, S. Shtrikman, J. Mech. Phys. Solids 10 (1962) 335.

[22] R. Hill, Proc. Phys. Soc Lond. A65 (1952) 349.

[23] O. Mercier, K.N. Melton, G. Gremaud, J. Haegi, J. Appl.
Phys. 51 (3) (1980) 1833.

[24] T.W. Clyne, P.J. Withers, An Introduction to Metal Matrix Composites, Cambridge University Press, Cambridge, 1993.

[25] R. Chang, L.J. Graham, J. Appl. Phys. 37 (1966) 3778.

[26] C.M. Jackson, H.J. Wagner, R.J. Wasilewski, NASA-SP 5110 (1972) 49.

[27] J. Shackelford, W. Alexander, The CRC Materials Science and Engineering Book, CRC Press, Boca Raton, 1992, pp. 358.

[28] T. Saburi, S. Nenno, in: H.I. Aaronson, D.E. Laughlin, R.F. Sekerka, C.M. Wayman (Eds.), Solid-Solid Phase Transformations, The Metallurgical Society of AIME, Warrendale, 1981, p. 1455.

[29] M.A.M. Bourke, J.A. Goldstone, N. Shi, J.E. Allison, M.G. Stout, A.C. Lawson, Scripta Metall. Mater. 29 (1993) 771. 